\title{
Loss of Shp2 within radial glia is associated with cerebral cortical dysplasia, glial defects of cerebellum and impaired sensory-motor development in newborn mice
}

\author{
YU ZHU $^{1 *}$, JIAN SHEN $^{1 *}$, TIANFU SUN $^{1 *}$, HAO JIANG $^{1}$, KANGLI XU $^{1}$, THITI SAMUTHRAT ${ }^{1}$, \\ YICHENG XIE $^{2}$, YUXIANG WENG ${ }^{1}$, YONGDA LI ${ }^{1}$, QIANGMIN XIE ${ }^{3}$ and RENYA ZHAN ${ }^{1}$ \\ ${ }^{1}$ Department of Neurosurgery, The First Affiliated Hospital, Zhejiang University School of Medicine, \\ Hangzhou, Zhejiang 310003, P.R. China; ${ }^{2}$ Department of Psychiatry, Kinsmen Laboratory of \\ Neurological Research and Brain Research Centre, University of British Columbia, Vancouver, BC V6T 1Z3, \\ Canada; ${ }^{3}$ Zhejiang Respiratory Drugs Research Laboratory of China Food and Drug Administration, \\ Laboratory Animal Center of Zhejiang University, School of Medicine, Hangzhou, Zhejiang 310058, P.R. China
}

Received March 22, 2017; Accepted September 20, 2017

DOI: $10.3892 / \mathrm{mmr} .2017 .8236$

\begin{abstract}
Radial glia are key neural progenitors involved in the development of the central nervous system. Tyrosine-protein phosphatase non-receptor type 11 (Shp2) is a widely expressed intracellular enzyme with multiple cellular functions. Previous studies have revealed the critical role of Shp2 in a variety of neural cell types; however, further investigation into the function of Shp2 within radial glia is required. In the present study, a conditional knockout mouse was generated using a human glial fibrillary acidic protein (hGFAP)-Cre driver, in which the Shp2 genes were deleted within radial glia. Loss of Shp2 within radial glia was associated with developmental retardation, postnatal lethality, reduced brain size and thinner cerebral cortices in newborn mice. Deletion of Shp2 also led to an increase in gliogenesis, a reduction in neural genesis and extracellular signal-regulated kinase signaling within the cerebral cortex. Furthermore, glial cell defects within the cerebellum of Shp2 mutants were observed, with abnormal granular cell retention and glial cell alignment in the external granular layer. In addition, Shp2 mutants exhibited impaired sensory-motor development. The results of the present study
\end{abstract}

Correspondence to: Professor Renya Zhan, Department of Neurosurgery, The First Affiliated Hospital, Zhejiang University School of Medicine, 79 Qingchun Road, Hangzhou, Zhejiang 310003, P.R. China

E-mail: 06yxsyzy@163.com

*Contributed equally

Abbreviations: CKO, conditional knockout; EGL, external granular layer; hGFAP, human glial fibrillary acidic protein; IGL, internal granular layer; VZ, ventricular zone; WT, wild-type

Key words: radial glia, Shp2, cerebral cortex, cerebellum, sensory-motor function suggested that Shp2 may have an important role within radial glia, and regulate cerebral cortical and cerebellar development in newborn mice.

\section{Introduction}

Tyrosine-protein phosphatase non-receptor type 11 (Shp2) is a ubiquitously expressed intracellular enzyme which has critical cellular functions in a variety of processes, including survival, proliferation and differentiation $(1,2)$; however, systemic Shp2 loss has been associated with embryonic lethality (3).

Shp2 has been demonstrated to regulate numerous signaling events, among which the mitogen-activated protein kinase (MAPK) signaling pathway was identified to be commonly facilitated by Shp2. Mutations in the protein tyrosine phosphatase, non-receptor type 11 (PTPN11) gene, which encodes Shp2 protein, have been reported to inhibit MAPK signaling $(4,5)$. Due to its pivotal role, investigations into the effects exhibited by Shp 2 have been conducted using Shp 2 conditional knockout (cKO) mouse models in a variety of neural tissues and cell types using distinct Cre recombinase drivers. These models revealed that Shp2 may have roles within numerous areas and cell types during brain developmental processes, including forebrain neuron development (6), cerebellum foliation (7) and oligodendrocyte generation (8). Radial glia are neural progenitors with long radial processes, which serve key roles in the development of the central nervous system. The properties of radial glia were identified due to similarities with astrocytes, which contain glycogen granules and express glial fibrillary acidic protein (GFAP). In addition, radial glia have been considered to support the migration of nascent neurons (9); however, in the previous decade, investigations have focused on the progenitor capacity of radial glia within the cerebral cortex $(10,11)$ and cerebellum $(11,12)$.

In the present study, transgenic mice with hGFAP-Cre under the control of a glial-specific promoter were used to ablate Shp2 expression within radial glia $(13,14)$. In contrast to the GFAP-Cre system, which is commonly used to generate 
astrocyte-specific gene knockout model, the hGFAP-Cre recombinase is expressed within cerebral cortical radial glia at E13.5-E14 and cerebellar radial glia at E13.5-E16.5 $(15,16)$. Shp2 knockout within radial glia was associated with postnatal growth failure, cerebral cortical dysplasia with decreased extracellular signal-regulated kinase (ERK) signaling, glial defects of cerebellum and impaired sensory-motor functions. Collectively, the findings of the present study revealed the critical function of Shp2 in radial glia that contributes to cerebral cortical and cerebellar development in newborn mice.

\section{Materials and methods}

Mice. A total of 290 newborn mice were used in this study. The newborn mice as well as their parents had continuous access to food and water and were housed in cages in a room maintained at a temperature of $20-22^{\circ} \mathrm{C}$ with a 12 -h light/dark cycle. The present study was approved by the Ethics Committee the First Affiliated Hospital, Zhejiang University School of Medicine (Hangzhou, China). All mice received humane care, in accordance with the guide prepared by the Committee of Care and Use of Laboratory Animals. The hGFAP-Cre line and floxed Shp2 line were both purchased from the Jackson Laboratory (Ben Harbor, ME, USA). hGFAP-Cre line (17) were interbred with the floxed Shp2 line (18) to generate Shp2 $2^{\text {f/f; hGAFP }}$ Cre/+ mice, in which the Shp2 gene was conditionally knocked out (Shp2 CKO).

Western blot analysis. Whole brain and microdissected cortices lysates obtained from 14-day-old male newborn mice were extracted with radioimmunoprecipitation assay buffer (Biyuntian Biotechnology Co., Ltd., Haimen, China) containing $1 \mathrm{mM}$ phenylmethylsulfonyl fluoride (Haoxin Biotechnology, Hangzhou, China) (http://www.hzhxbio. com). Protein concentration was measured using a bicinchoninic acid protein assay kit (CW Biotech, Beijing, China). A total of $40 \mu \mathrm{g} /$ lane protein was separated by $10 \%$ SDS-PAGE, transferred onto nitrocellulose membranes (EMD Millipore, Billerica, MA, USA), and then incubated with $10 \%$ bovine serum albumin solution (Sigma-Aldrich; Merck KGaA, Darmstadt, Germany) at $37^{\circ} \mathrm{C}$ for $1 \mathrm{~h}$. Blots were probed with antibodies specific for Shp2 (sc-7384, 1:1,000; Santa Cruz Biotechnology, Inc., Dallas, TX, USA), phosphorylated ERK (pERK, 4370S, 1:2,000; Cell Signaling Technology, Inc., Danvers, MA, USA), total ERK (tERK, 9102S, 1:1,000), pAKT serine/threonine kinase (pAKT, 4060S, 1:2,000) and total AKT (tAKT, 4685S, 1:1,000) (all from Cell Signaling Technology, Inc.) and $\beta$-tubulin (sc-365791, 1:500; Santa Cruz Biotechnology, Inc.) overnight at $4^{\circ} \mathrm{C}$. Membranes were then probed with goat anti-rabbit 800 (SA5-35571, 1:10,000; Invitrogen; Thermo Fisher Scientific, Inc., Waltham, MA, USA) or anti-mouse antibodies (A-11001, 1:5,000; Invitrogen; Thermo Fisher Scientific, Inc.) for $2 \mathrm{~h}$ at room temperature. Immunoreactive bands were visualized using a two-color infrared imaging system (LI-COR Biosciences, Lincoln, NE, USA).

Histology and immunohistochemistry. Newborn mice were anesthetized with pentobarbital sodium salt (Sigma-Aldrich; Merck $\mathrm{KGaA}$ ) and transcardially perfused with $0.9 \% \mathrm{NaCl}$, followed by 3 or $4 \%$ paraformaldehyde/PBS. The cerebrum and cerebellum were removed, fixed overnight with PBS ( $\mathrm{pH} 7.2$ ) containing $4 \%$ paraformaldehyde at $4{ }^{\circ} \mathrm{C}$ and embedded in paraffin wax. Samples were cut into $4 \mu \mathrm{m}$ sections. Hematoxylin and eosin (H\&E) staining (Beyotime Institute of Biotechnology, Haimen, China) and toluidine blue staining (Wuhan Goodbio Technology Co., Ltd., Wuhan, China) (http://www.servicebio.cn) were performed according to the manufacturer's instructions. Following deparaffinization, tissues were rehydrated with graded alcohol (100, 95 and $50 \%$ ) and blocked with goat serum (Fuzhou Maixin Biotech Co., Ltd., Fuzhou, China) at $37^{\circ} \mathrm{C}$ for $15 \mathrm{~min}$. Antigen retrieval was performed using $0.01 \mathrm{M}$ citrate buffer ( $\mathrm{pH} 6.0$ ) at $100^{\circ} \mathrm{C}$ for $2 \mathrm{~min}$, and then washed with PBS. Permeablization was performed for 10 min with PBS containing $0.1 \%$ Triton $\mathrm{X}-100$ and then washed with PBS. Incubation of primary and secondary antibodies for immunostaining was performed according to standard protocols. The primary antibodies were incubated overnight at $4^{\circ} \mathrm{C}$ using the following: Anti-GFAP (HPA056030, 1:100; Sigma-Aldrich; Merck KGaA), anti-neuronal specific nuclear protein (NeuN, ABN78, 1:200; EMD Millipore), anti-Ki67 (ab15580, 1:100; Abcam, Cambridge, UK), anti-ERK (4696S, 1:100; Cell Signaling Technology, Inc.), anti-pERK (9101S, 1:250; Cell Signaling Technology, Inc.), anti-AKT (4685S, 1:100; Cell Signaling, MA, USA), anti-pAKT (4060S, 1:100; Cell Signaling Technology, Inc.). Then incubation of secondary antibodies at room temperature for $2 \mathrm{~h}$ was performed with Goat anti-rabbit 800 (SA5-35571, 1:100; Invitrogen; Thermo Fisher Scientific, Inc.) or anti-mouse antibodies (A-11001, 1:100; Invitrogen; Thermo Fisher Scientific, Inc.). Terminal deoxynucleotidyl-transferase-mediated dUTP nick end labeling (TUNEL) staining was performed according to the manufacturer's protocol (Roche Applied Science, Branford, CT, USA). Images were collected with a laser confocal microscope (Olympus IX71; Olympus Corporation, Tokyo Japan), or the Nikon E600FN (Nikon Corporation, Tokyo, Japan) microscope and 5 randomly selected fields of view were observed.

Cell counting was performed according to a previously published method (19). Quantification of cell numbers was performed using the Image J software version 1.48 (National Institutes of Health, Bethesda, MD, USA) by a blinded observer.

Newborn mice behavior analyses. Behavior of newborn mice aged 5-15 days was examined as described in a recent study (20) with modifications. General health of the newborns was evaluated by measuring body weight. For motor and sensory reflex development evaluation, the righting reflex test, sound attraction test, the wire hanging test and nest finding test were performed as follows.

Righting reflex test. Newborn mice were placed on their backs and the duration for righting itself on all four limbs was measured. 'Success' was defined as the duration of righting in $<3 \mathrm{sec}$.

Sound attraction test. Each newborn was placed at the center of a round platform with a radius of $15 \mathrm{~cm}$ with soft walls to provide protection against edges. Auditory stimuli were administered by 
A

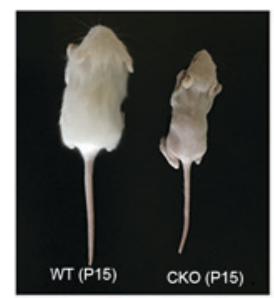

D

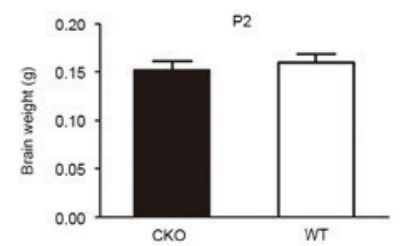

B
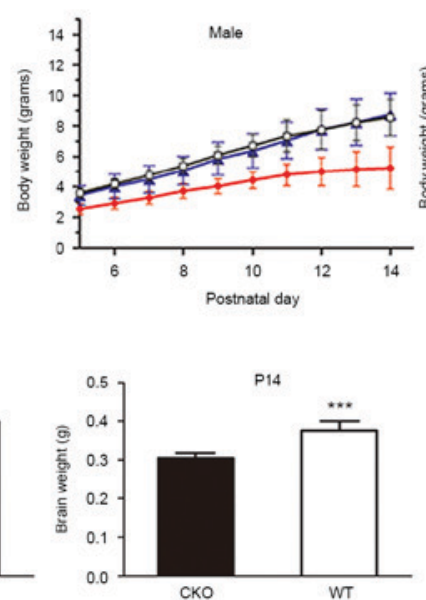

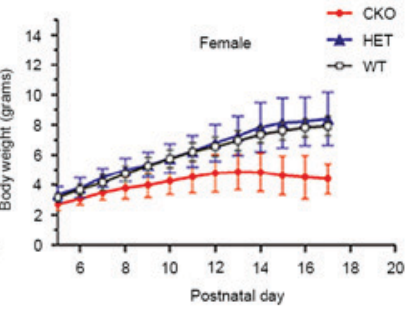

E

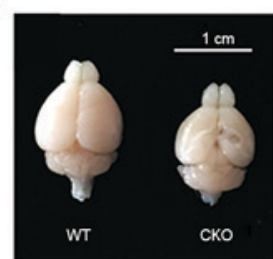

C

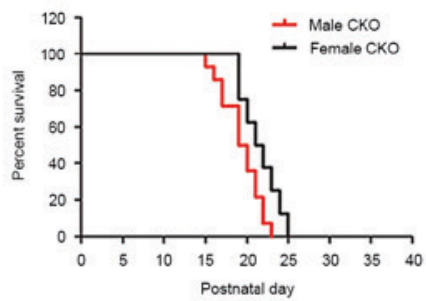

F

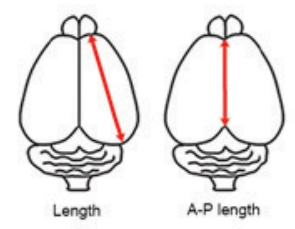

G

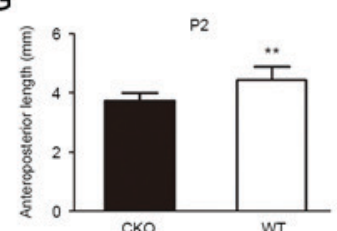

$\mathrm{H}$

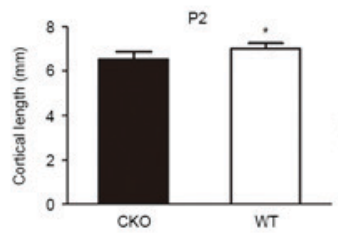

I
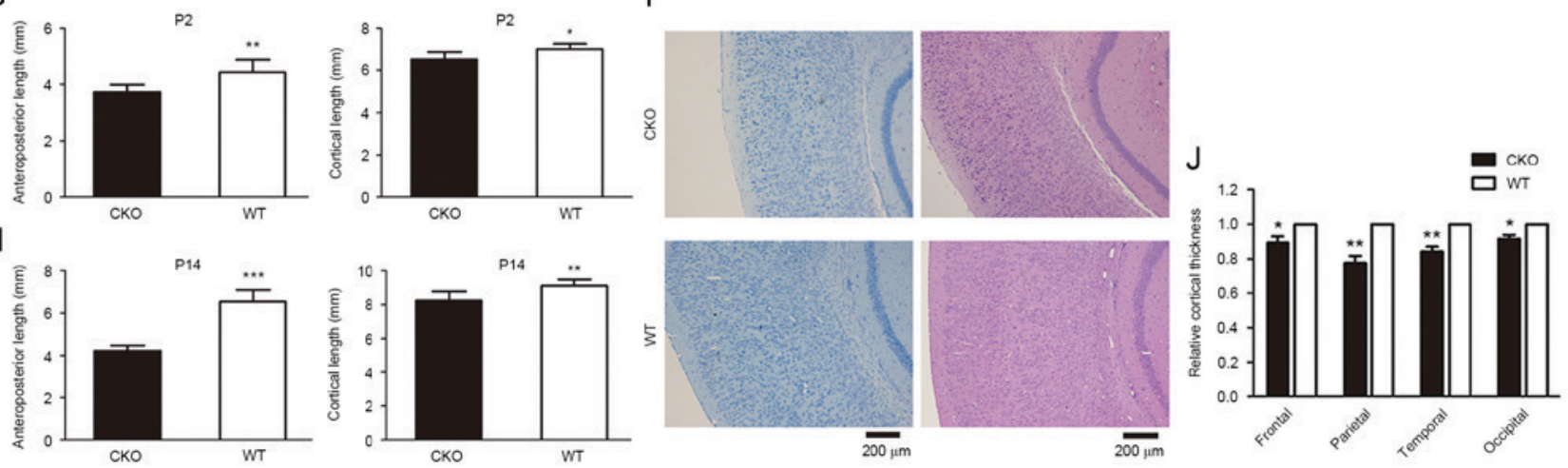

Figure 1. Loss of Shp2 in radial glia leads to postnatal growth retardation, defects in cerebral development and lethality within newborn mice. (A) Gross appearance of a representative pair of WT and CKO male littermates at P15. (B) Male and female body weight growth of newborn mice. (C) Postnatal lethality of Shp2 CKO male mice, mean survival days=19.4 and median survival days=19.5. Female mice, mean survival days=21.6 and median survival days=21.5. (D) Whole brain weights of Shp2 CKO and Shp2 WT mice at P2 and P14. ${ }^{* * *} \mathrm{P}<0.001$ vs. CKO. (E) Whole brains of Shp2 CKO and WT mice at P14. (F) Measurements of cortical parameters. Shp2 CKO mice revealed a decrease in A-P length and cortical length at (G) P2 and (H) P14 compared with in Shp2 WT mice. ${ }^{*} \mathrm{P}<0.05,{ }^{* *} \mathrm{P}<0.01,{ }^{* * *} \mathrm{P}<0.001$ vs. CKO. (I) Hematoxylin and eosin and toluidine blue staining of coronal sections of Shp2 CKO and WT brains at P14. Scale bar, $200 \mu \mathrm{m}$. (J) Quantitative analysis of cortical thickness. $\mathrm{P}<0.05,{ }^{* *} \mathrm{P}<0.01$. Data are expressed as the mean \pm standard deviation. Panel $\mathrm{B}, \mathrm{n}=4$; panel C, n=14; panel D, n=6; panel E, n=6; panel F, P=6; panel J, n=5. A-P length, anteroposterior length; CKO, conditional knockout; Shp2, tyrosine-protein phosphatase non-receptor type 11; WT, wild-type; HET, heterozygous.

gentle scratch to sandpaper and the response of the newborn was measured. A score of 0 indicated no response, 0.5 was given when the newborn turned his head to the direction of the sound and a score of 1 was given when the newborn moved towards the sound on three consecutive accounts.

Wire hanging test. The duration the newborn can hold on a vertical wire before landing on a soft material was measured. The mean time was recorded for three sequential times.

Nest finding test. The ability for the newborn to return to the nest was measured by placing the newborn $2 \mathrm{~cm}$ from the nest and observed for $60 \mathrm{sec}$. Mice behavior was scored 0 when no attempt to return to the nest was observed. A score of 0.5 was given when the newborn successfully made to the nest or moved in the correct direction towards the nest at least once and a score of 1 was given when the newborn successfully arrived the nest on three consecutive accounts.

Statistical analysis. Statistical analyses were conducted using either two-way analysis of variance with the Bonferroni's post hoc test or the Student's t-test to compare groups. All experiments were repeated $\leq 4$ times. Data are presented as the mean \pm standard deviation. $\mathrm{P}<0.05$ was considered to indicate a statistically significant difference.

\section{Results}

Deletion of Shp2 in radial glia leads to postnatal growth failure and lethality in newborn mice. Shp $2^{\mathrm{f} / \mathrm{f}}$ mice were crossed with hGFAP-Cre mice to produce litters of +/+ Shp2 wild-type (WT), -/+ heterozygous Shp2 KO and -/- Shp2 CKO mice. Successful deletion of Shp2 in the radial glia was validated on both mRNA and protein levels (data not shown). Retardation in postnatal growth and reductions in body weight were observed in the Shp2 CKO group compared with the WT mice in the first two weeks after birth; alterations in postnatal weight of heterozygous mice were not observed (Fig. 1A and B). The results of the present study suggested that heterozygous Shp2 KO was not associated with a change in postnatal weight. In addition, postnatal lethality exhibited by Shp2 CKO mice appeared to be associated with sex, Shp2 
A

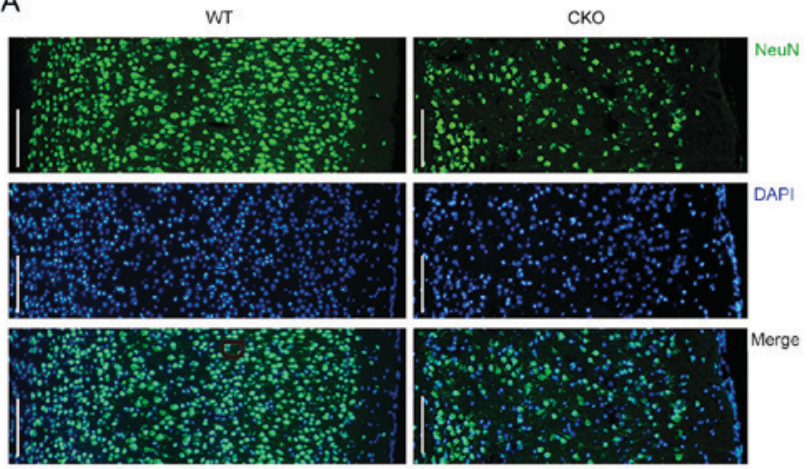

B

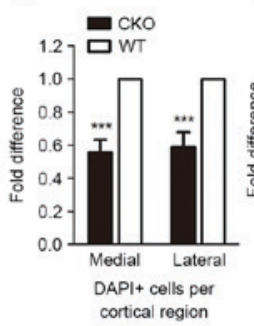

C

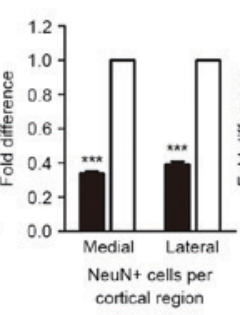

$\mathrm{D}$

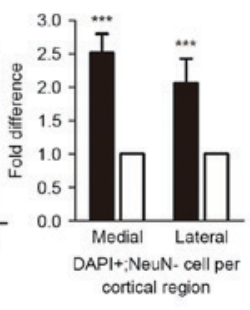

E GFAP DAPI

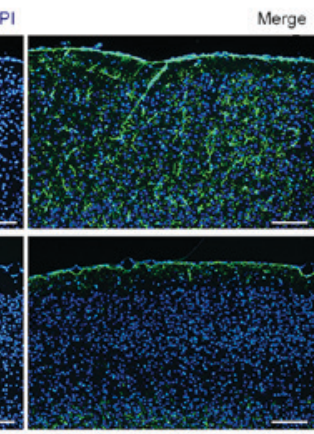

F
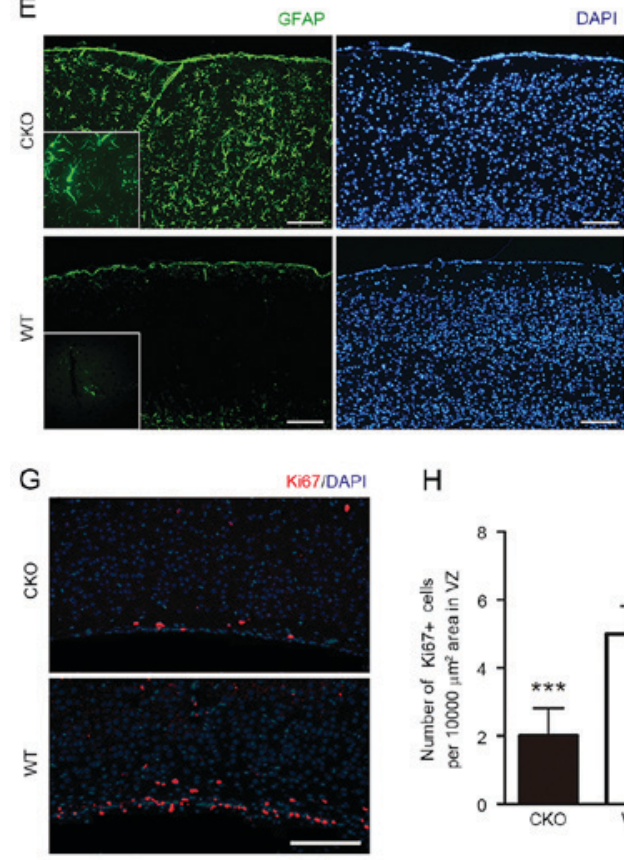

$\mathrm{H}$
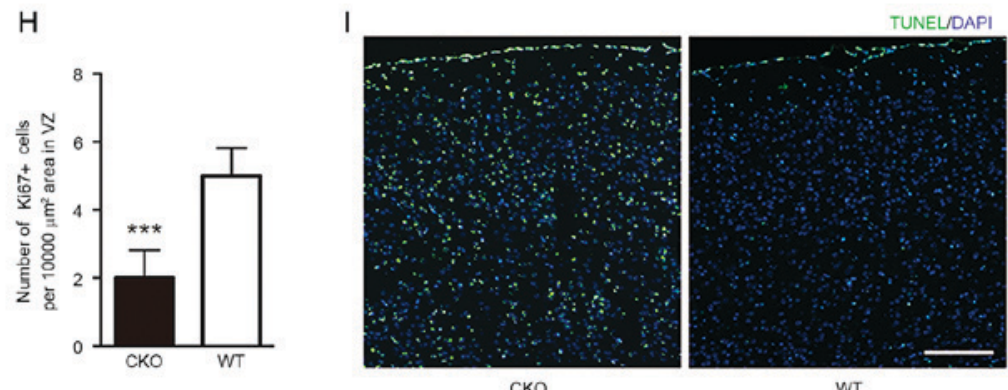

CKO

WT

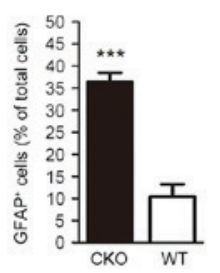

Figure 2. Loss of Shp2 in radial glia leads to altered cellular composition of the cortex, impaired corticogenesis and decreased cortical ERK signaling. (A) Coronal sections of Shp2 CKO and Shp2 WT cortices at P14 were immunostained with anti-NeuN and counterstained with DAPI. Total cell number per cortical region from the VZ to the pial surface was calculated by counting (B) $\mathrm{DAPI}^{+}$and (C) $\mathrm{NeuN}^{+}$cells in the primary motor cortex and the primary somatosensory cortex, presented as the medial and lateral sections, respectively. (D) Fold difference of DAPI ${ }^{+}$and $\mathrm{NeuN}^{+}$cell in the primary motor cortex and primary somatosensory cortex, presented as the medial and lateral sections. ${ }^{* * *} \mathrm{P}<0.001$ vs. WT. Scale bar, $200 \mu \mathrm{m}$. (E) Coronal sections of cortices from Shp2 CKO and Shp2 WT mice at P14 were immunostained with GFAP and counterstained with DAPI. (F) Corresponding total GFAP' cell number from the VZ to the pial surface. ${ }^{* * *} \mathrm{P}<0.001$ Scale bar, $200 \mu \mathrm{m}$. (G) Immunostaining revealed Ki67+ cells within the VZ tissue of Shp2 CKO and Shp2 WT mice tissue. (H) Compared with in the Shp2 WT group, the number of Ki67+ cells were significantly reduced. ${ }^{* * *} \mathrm{P}<0.001$ vs. WT. (I) Cell apoptosis was analyzed with a TUNEL assay at P14. Scale bar, $200 \mu \mathrm{m}$. CKO, conditional knockout; WT, wild-type; NeuN, neuronal specific nuclear protein; GFAP, glial fibrillary acidic protein; Shp2, tyrosine-protein phosphatase non-receptor type 11; VZ, ventricular zone; TUNEL, terminal deoxynucleotidyl transferase dUTP nick end labelling.

CKO male mice were affected more severely compared with in Shp2 CKO female mice (Fig. 1C; average lifetimes: Male 19.36 days, female 21.63 days; $\mathrm{P}=0.042$, Student's t-test).

Shp2 CKO mice exhibit cerebral cortical development defects. A previous study in which Erk2 was conditionally deleted in radial glia resulted in smaller brain size and defective cortical cytoarchitecture (19). As Shp2 is an important modulator of the MAPK signaling (21), the effects of Shp2 knockout may be associated to the effects observed with in Erk2 CKO mice. The total brain weight was similar in Shp2 CKO group and the WT group at P2, but was significantly lower in Shp2 CKO group compared with WT mice at P14 (Fig. 1D). Shp2 CKO mice also exhibited a decrease in brain size (Fig. 1E), mainly caused by significant decrease in cortical area measured by anteroposterior length and cortical length compared with WT at P2 and P14 (Fig. 1F-H). Further analysis of cortical thickness within the Shp2 CKO mice indicated a significant reduction in cortical thickness (Fig. 1I) compared with in the Shp2 WT group. In addition, a reduction in thickness within primary motor, somatosensory, auditory and visual cortices was observed in CKO mice compared with WT (Fig. 1J). The results of the present study indicate that deletion of Shp2 in radial glia led to defective cortical development.

Loss of Shp2 within radial glia leads to altered cellular composition of the cortex and impaired corticogenesis. To investigate the cellular basis for the reduction in cortical thickness within Shp2 CKO mice, the total number of DAPI ${ }^{+}$and $\mathrm{NeuN}^{+}$cells per cortical region were quantified. The results revealed a $>40 \%$ reduction in total cell density within the Shp2 

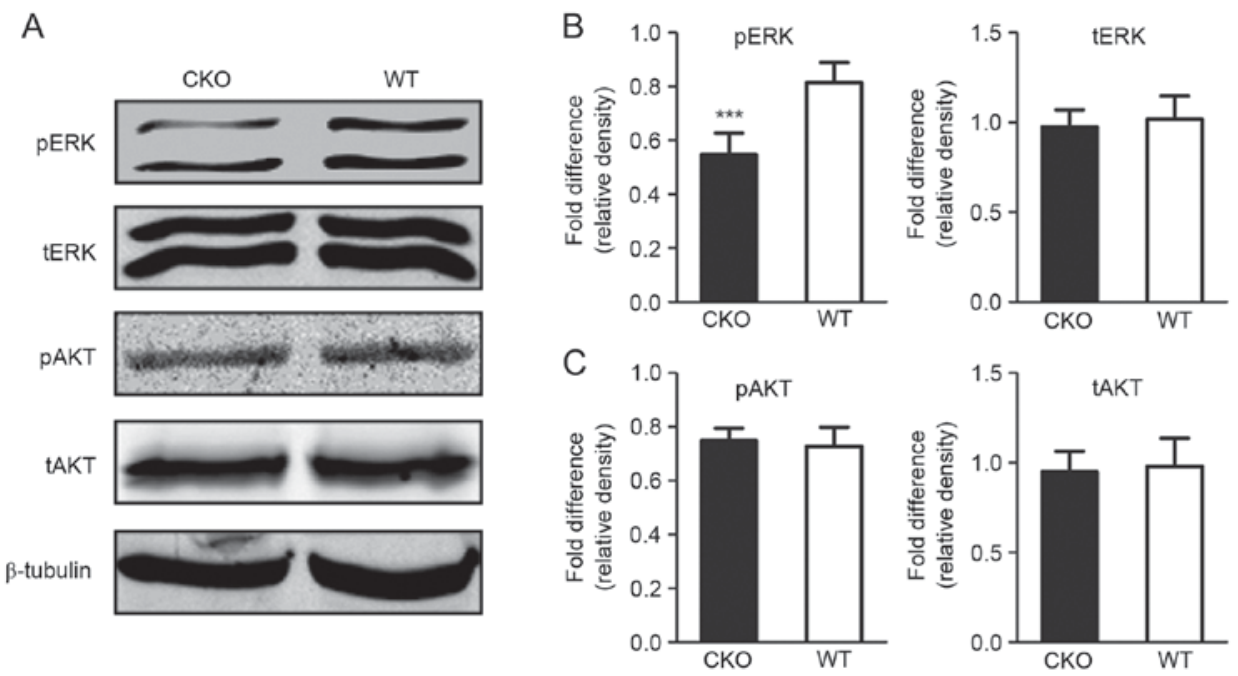

Figure 3. ERK activity is altered within Shp2 CKO microdissected cerebral cortex tissue. (A) Western blot analysis of cortical lysates isolated from P8 newborn mice within the Shp2 CKO and WT groups. (B) Shp2 CKO mice revealed a significant decrease in pERK expression levels, but no change in tERK expression level compared with Shp2 WT mice at P8; ${ }^{* * *} \mathrm{P}<0.001$. (C) A significant alteration in the expression levels of pAKT and tAKT within the Shp2 CKO was not observed compared with those in the Shp2 WT group. Data are expressed as the mean \pm standard deviation, $\mathrm{n}=4-8$ in each group. CKO, conditional knockout; ERK, extracellular signal-regulated kinase; p, phosphorylated; t, total; AKT, AKT serine/threonine kinase; Shp2, tyrosine-protein phosphatase non-receptor type 11; WT, wild-type.

CKO cortex (Fig. 2A and B). In addition, a significant decrease, $>60 \%$, of $\mathrm{NeuN}^{+}$cells per cortical region and a $>2$-fold increase in non-neuronal cells was detected in Shp2 CKO mice compared with WT mice (Fig. 2C and D). The results of the present study indicated that reduced cortical thickness due to Shp2 deletion within radial glia was associated with the generation of fewer neurons and more non-neuronal cells. Immunohistochemistry analysis of glial cell markers led to the identification of non-neuronal cells; a marked increase in $\mathrm{GFAP}^{+}$glial cells was observed throughout the cortex in CKO mice compared with WT mice (Fig. 2E and F). A previous study reported that radial glia may regulate gliogenesis and neurogenesis within the mammalian cerebral cortex (11); the present study suggested that Shp2 may serve a role in radial glial cell differentiation within the process of gliogenesis, but inhibits neurogenesis.

The proliferation marker Ki67 in the ventricular zone (VZ) of mice was analyzed at P4 in the present study. A significant reduction of $\mathrm{Ki}^{+} 7^{+}$cells was observed in the Shp2 CKO group compared with in the Shp2 WT group (Fig. 2G and H), indicating that neural stem cell proliferation in the cerebral cortex was impaired within Shp2 CKO mice. Cell survival was also evaluated within Shp2 CKO cortex tissue via TUNEL analysis. An increase in apoptosis was observed at P14 within the Shp2 CKO mice compared with WT (Fig. 2I). The findings of the present study indicated that the reduction in cell density exhibited within the Shp2 CKO group may be due to a decrease in cell proliferation and an increase in cell apoptosis.

Erk activity is altered in the Shp2 CKO newborn mice. Shp2 has been previously demonstrated to be an important factor involved in a variety of signaling cascades, among which ERK and AKT are two effector molecules investigated in studies where Shp2 is dysregulated (21). Phenotypic alterations within the cerebral cortex of Shp2 CKO mice were similar to those described in a previous study of mice with defective ERK signaling within the radial glia (19). Analyses of tERK, pERK, tAKT and pAKT expression levels revealed a decrease in pERK levels within the cerebral cortex of Shp2 CKO mice compared with in Shp2 WT mice; however, significant alterations in tERK, tAKT and pAKT levels were not observed (Fig. 3A-C). These results suggested that the ERK signaling pathway within the cerebral cortex is affected by Shp2 KO in radial glia.

Deletion of Shp2 in radial glia leads to glial defects in cerebellum. Radial glia possess the ability to generate glial and neuronal cells within the cerebral cortex. A previous study demonstrated that the majority of radial glia bypass neurogenesis and retain the glial cell phenotype within the cerebellum (22), similar to the gross appearance of cerebellum tissue of the Shp2 CKO mice (Fig. 1E). However, histological analysis by $\mathrm{H} \& \mathrm{E}$ staining of cerebellar sections indicated that at P14 in the Shp2 CKO cerebellum there were abnormal cell clusters at the pial surface, which is the site of the neonatal transient external granular layer (EGL), while in WT this outer region was not present (Fig. 4A and B). Furthermore, immunohistochemical analysis of cerebellar sections fromP14 mice showed aberrant alignment of the glial cells in the EGL of Shp2 CKO mice (Fig. 4C and D). As radial glia-derived glial cells in the EGL have critical functions in development of cerebellum by mediating granular cell migration from EGL to the internal granular layer (IGL). These results suggest that Shp2 deficiency in radial glia resulted in defective glial cells in the EGL, which led to failed migration of granular cells and abnormal retention of cell clusters. Together, these data suggest that expression of Shp2 in the radial glia has a pivotal role in regulating cerebellar development.

Deletion of Shp2 in radial glia is associated with impaired sensory-motor development. The emergence of coordinated sensory-motor behaviors are dependent upon the establishment 
A
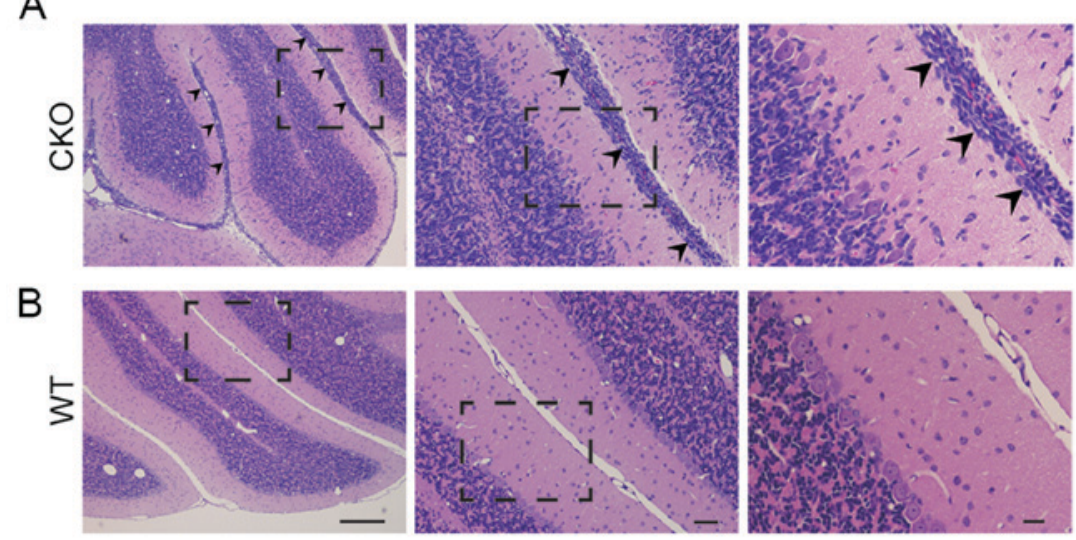

C

GFAP

DAPI

Merge
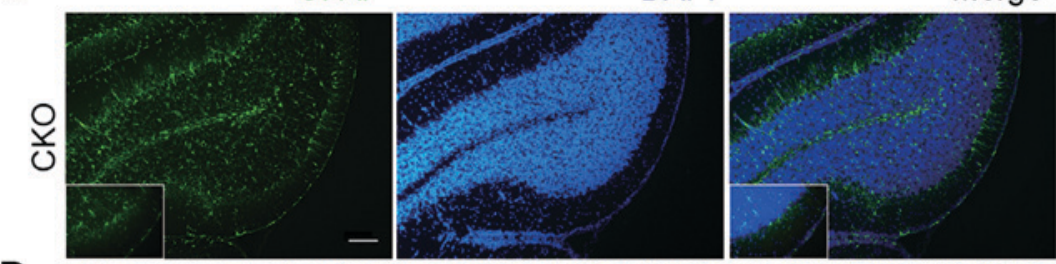

$\mathrm{D}$
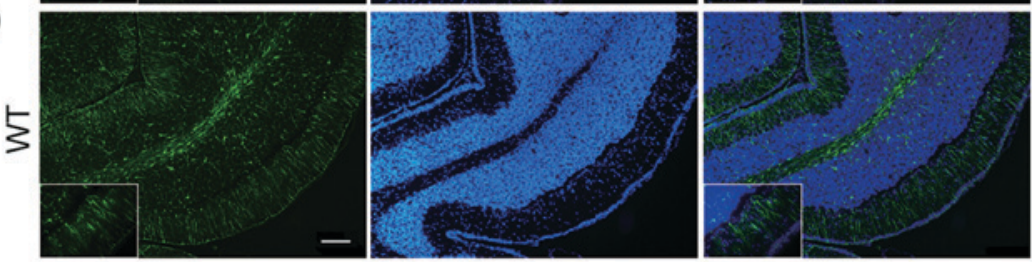

Figure 4. Deletion of Shp2 in radial glia leads to glial defects in cerebellum. Hematoxylin and eosin staining of cerebellar sections from (A) Shp2 CKO and (B) Shp2 CKO mice at P14 revealed the presence of abnormally retained cell clusters at the pial surface of the external granular layer of the Shp2 CKO cerebellum. Arrow heads represent retained cell clusters. Boxes in left/middle panels represent areas further enlarged in the panel on their respective right side. Scale bars (left to right), 200, 50 and $20 \mu \mathrm{m}$. Immunostaining of cerebellum tissue of (C) Shp2 CKO and (D) Shp2 WT mice with anti-GFAP antibodies. The inset boxes are enlargements of EGL, showing aberrant alignment of the glial cells in Shp2 CKO mice at P14. Scale bar, $200 \mu \mathrm{m}$. CKO, conditional knockout; GFAP, glial fibrillary acidic protein; Shp2, tyrosine-protein phosphatase non-receptor type 11; WT, wild-type; EGL, external granular layer.

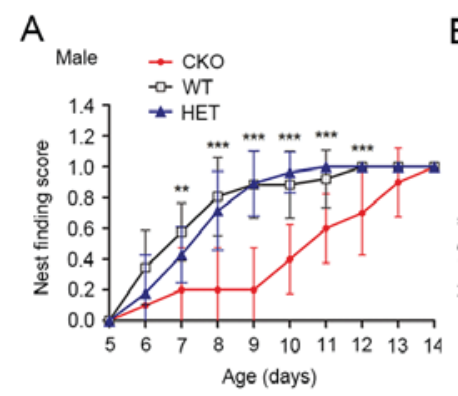

E

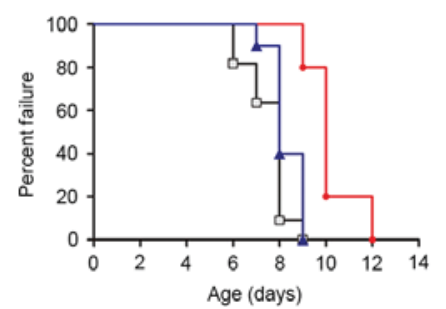

B

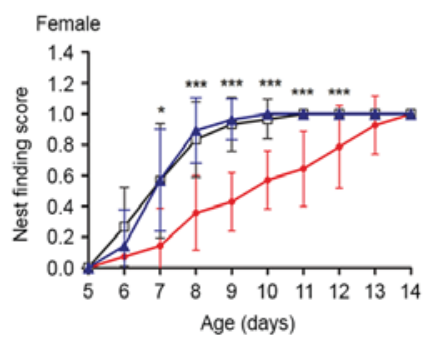

$\mathrm{F}$

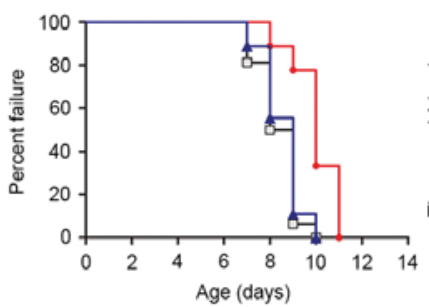

C

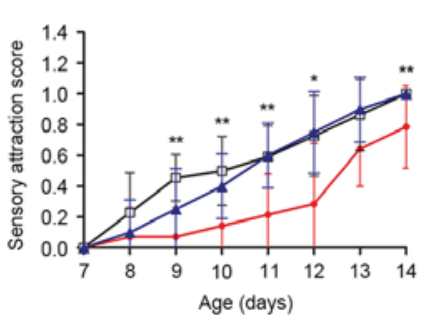

G

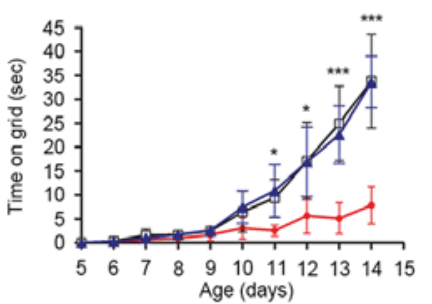

D

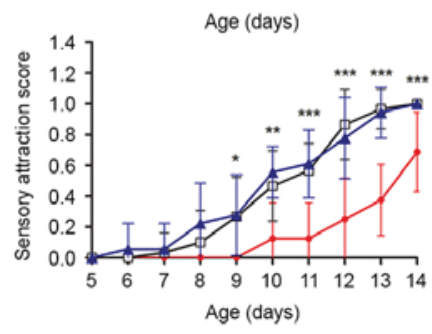

$\mathrm{H}$

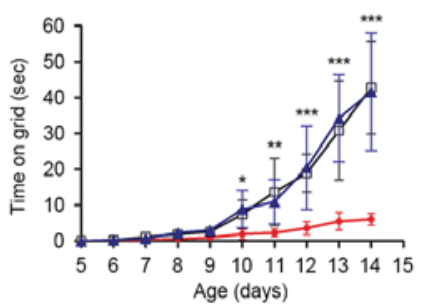

Figure 5. Deletion of Shp2 in radial glia impairs sensory-motor development. Nest finding score of (A) male and (B) female Shp2 CKO, HET and WT newborn mice. Scores are represented on a scale from 0 , no response, to 1 , success arrival to the nest in three trials. Sensory attraction score of (C) male and (D) female newborn mice. Scores are represented on a scale from 0 , no response, to 1, moved towards the stimulus in three trials. Righting reflex of (E) male and (F) newborn mice. 'Success' was defined as the day on which newborns achieved the criteria of righting in $<3$ sec. Wire hanging ability of (G) male and $(\mathrm{H})$ female newborn mice. Duration was measured until the newborn release their hold of a vertical wire. Data are expressed as the mean \pm standard deviation. CKO and HET groups were compared. ${ }^{*} \mathrm{P}<0.05,{ }^{* * *} \mathrm{P}<0.01,{ }^{* * *} \mathrm{P}<0.001$ CKO vs. HET groups. Shp2, tyrosine-protein phosphatase non-receptor type 11 ; CKO, conditional knockout; HET, heterozygous; WT, wild-type. 
of distinct connections between the discrete populations of functioning neurons within the cortex and cerebellum $(23,24)$. Patients with mutations in the PTPN11 gene associated with Noonan syndrome exhibit numerous sensory and motor disorders, including hearing loss, muscle hypotonia, motor development delay and learning disabilities (25). Shp2 CKO mice exhibited marked ataxia at P14. As cerebral cortical and cerebellar development was observed to be severely affected by Shp2 loss, the effect of Shp2 CKO for sensory-motor function development of newborn mice was investigated. The nest finding test was applied to evaluate the comprehensive ability of newborn mice to identify and return to the nest using motor skills and motivation. The results of the present study demonstrated a decrease in nest finding scores in the Shp2 CKO group compared with in the WT and heterozygous groups from P7 in both sexes; however, all mice achieved maximal scores at postnatal day 14 (Fig. 5A and B). The capability of newborn mice to react to auditory stimulus was investigated $\sim 2$ days after the nest finding test; development of this ability within Shp2 CKO mice of both sexes were delayed compared with in the Shp2 WT and Shp2 heterozygous mice groups from P9; the marked variation remained evident at P14 (Fig. 5C and D). Muscle strength of the newborn mice was assessed via the wire hanging and righting reflex tests. In both sexes, Shp2 CKO mice demonstrated a delayed acquisition of righting ability from P9 (Fig. 5E and F) and a significant reduction in wire hanging duration from P11 (Fig. 5G and H) compared with in the WT and heterozygous groups. The results of the present study suggested that Shp2 expression in radial glial is involved in the development of somatosensory function and motor function in newborn mice.

\section{Discussion}

The size and complexity of cerebral cortex in mammals has been demonstrated to be developmentally associated with the generation of neural progenitor cells $(26,27)$; structural development of the cerebral cortex has been reported to be regulated by a variety of signaling molecules and transcription factors $(28,29)$. In the present study, newborn mice deficient of Shp2 in radial glia, the key organizer and progenitor cells in cerebral cortex, exhibited growth retardation and postnatal lethality accompanied with significant defects in the cerebral cortex. Smaller and thinner cerebral cortices were associated with decreased cortical cell density, reduced proliferative ability of VZ neural progenitors and increased cortical cell apoptosis. As radial glia are considered to be the primary progenitor cell type that comprise the majority of mitotically active cells within the VZ (30), the results of the present study may indicate that Shp2 is indispensable for the self-renewal and proliferation of cortical radial glia. In addition, appropriate functioning of the cerebral cortex depends on accurate regulation of the generation of various types of neural cells, in particular neurons and glia $(31,32)$. Almost all neurogenesis occurs prenatally, except in the subventricular and subgranular zones; however, the generation of glia predominantly perinatally or in the early postnatal period. The sequential processes of neuro- and gliogenesis are required for normal cytoarchitecture of the cerebral cortex $(33,34)$. The present study reported that Shp2 KO within radial glia resulted in the generation of fewer cortical neurons, but an increase in the number of glial cells. Therefore, Shp2 may affect the differentiation process of radial glia of the cerebral cortex.

A previous study demonstrated that the conditional deletion of Erk2 within radial glia using the hGFAP-Cre recombinase system was associated with cerebral deficits, analogous to the results of the present study, including reduced brain size and ventricular proliferative ability, as well as increased gliogenesis and decreased neurogenesis (19). ERK1/2 has been demonstrated to be a critical downstream factor of Shp2; ERK2 is 13 times more abundant than ERK1 in the superficial cortex of nude mice (35). Analysis of the ERK signaling pathway revealed a marked reduction in cortical pERK levels within Shp2 CKO mice compared with WT. The results of the present study supported the pivotal role of the ERK pathway in the regulation of corticogenesis. In addition, Shp2 may be involved in the regulation of radial glia proliferation and differentiation via modulation of ERK activity.

Unlike its role in the cerebral cortex, radial glia do not generate granular cells inn mammalian cerebellum (36); however, radial fibers develop from the radial glia and facilitate inward migration of granular cells from the EGL to the IGL post-birth (22). In the present study, abnormal retention of granular cells within the EGL was observed, accompanied with defective glial fibers between the EGL and the IGL. However, histological analysis did not reveal significant alterations in cell density of granular cells within the IGL of Shp2 CKO. The gross appearance of Shp2 CKO cerebellum was similar to that of Shp2 WT mice. These findings indicated that Shp2 expression in radial glia of the cerebellum is involved in the migration process of granular cells during cerebellar development; however, Shp2 function may be limited to the regulation of scaffolding within radial glia, with less influence on granular cell proliferation and maturation. However, the limited duration of observation ( $\sim 3$ weeks) may affect the observed effects of Shp2 CKO in further cerebellar lamination and foliation.

In the present study, developmental delay of sensory-motor function within Shp2 CKO mice was demonstrated in numerous aspects, including somatosensory function via the sound attraction test, muscle strength via the wire holding test and comprehensive sensory-motor function via the nest finding test. These results indicate a key role for radial glial Shp2 in the acquisition of complex neurological functions and behaviors. Additionally, these findings further suggested that radial glia may be an important neural cell type involved in human Noonan syndrome, associated with disease-associated sensory-motor dysfunctions and developmental deficits.

In conclusion, loss of Shp2 within radial glia resulted in cortical dysplasia with decreased ERK signaling. Shp2 CKO was associated with glial defects and impaired sensory-motor development of newborn mice. The results of the present study provide a critical insight into the function of radial glia Shp2 regulation of neural and behavioral development.

\section{Acknowledgements}

The present study was supported by the National Natural Science Foundation of China (grant no. 81371371, 2014). 


\section{References}

1. Grossmann KS, Rosario M, Birchmeier C and Birchmeier W: The tyrosine phosphatase Shp2 in development and cancer. Adv Cancer Res 106: 53-89, 2010.

2. Tajan M, de Rocca Serra A, Valet P, Edouard T and Yart A: SHP2 sails from physiology to pathology. Eur J Med Genet 58: 509-525, 2015.

3. Saxton TM and Pawson T: Morphogenetic movements at gastrulation require the SH2 tyrosine phosphatase Shp2. Proc Natl Acad Sci USA 96: 3790-3795, 1999.

4. Kontaridis MI, Swanson KD, David FS, Barford D and Neel BG PTPN11 (Shp2) mutations in LEOPARD syndrome have dominant negative, not activating, effects. J Biol Chem 281: 6785-6792, 2006.

5. Lajiness JD, Snider P, Wang J, Feng GS, Krenz M and Conway SJ: SHP-2 deletion in postmigratory neural crest cells results in impaired cardiac sympathetic innervation. Proc Natl Acad Sci USA 111: E1374-E1382, 2014.

6. Kusakari S, Saitow F, Ago Y, Shibasaki K, Sato-Hashimoto M, Matsuzaki Y, Kotani T, Murata Y, Hirai H, Matsuda T, et al: Shp2 in forebrain neurons regulates synaptic plasticity, locomotion, and memory formation in mice. Mol Cell Biol 35: 1557-1572, 2015.

7. Li K, Leung AW, Guo Q, Yang W and Li JY: Shp2-dependent ERK signaling is essential for induction of Bergmann glia and foliation of the cerebellum. J Neurosci 34: 922-931, 2014.

8. Zhu Y, Park J, Hu X, Zheng K, Li H, Cao Q, Feng GS and Qiu M: Control of oligodendrocyte generation and proliferation by Shp2 protein tyrosine phosphatase. Glia 58: 1407-1414, 2010.

9. Rakic P: Elusive radial glial cells: Historical and evolutionary perspective. Glia 43: 19-32, 2003.

10. Miyata T, Kawaguchi D, Kawaguchi A and Gotoh Y: Mechanisms that regulate the number of neurons during mouse neocortical development. Curr Opin Neurobiol 20: 22-28, 2010.

11. Barry DS, Pakan JM and McDermott KW: Radial glial cells: Key organisers in CNS development. Int J Biochem Cell Biol 46: 76-79, 2014.

12. Guo Z, Wang X, Xiao J, Wang Y, Lu H, Teng J and Wang W: Early postnatal GFAP-expressing cells produce multilineage progeny in cerebrum and astrocytes in cerebellum of adult mice. Brain Res 1532: 14-20, 2013.

13. Gan Q, Lee A, Suzuki R, Yamagami T, Stokes A, Nguyen BC, Pleasure D, Wang J, Chen HW and Zhou CJ: Pax6 mediates $\beta$-catenin signaling for self-renewal and neurogenesis by neocortical radial glial stem cells. Stem Cells 32: 45-58, 2014.

14. Way SW, McKenna J III, Mietzsch U, Reith RM, Wu HC and Gambello MJ: Loss of Tsc2 in radial glia models the brain pathology of tuberous sclerosis complex in the mouse. Hum Mol Genet 18: 1252-1265, 2009.

15. Zhuo L, Theis M, Alvarez-Maya I, Brenner M, Willecke K and Messing A: hGFAP-cre transgenic mice for manipulation of glial and neuronal function in vivo. Genesis 31: 85-94, 2001.

16. Wen J, Yang HB, Zhou B, Lou HF and Duan S: $\beta$-catenin is critical for cerebellar foliation and lamination. PLoS One 8: e64451, 2013.

17. Casper KB and McCarthy KD: GFAP-positive progenitor cells produce neurons and oligodendrocytes throughout the CNS. Mol Cell Neurosci 31: 676-684, 2006.
18. Li FF, Shen J, Shen HJ, Zhang X, Cao R, Zhang Y, Qui Q, Lin XX, Xie YC, Zhang LH, et al: Shp2 plays an important role in acute cigarette smoke-mediated lung inflammation. J Immunol 189: 3159-3167, 2012.

19. Samuels IS, Karlo JC, Faruzzi AN, Pickering K, Herrup K, Sweatt JD, Saitta SC and Landreth GE: Deletion of ERK2 mitogen-activated protein kinase identifies its key roles in cortical neurogenesis and cognitive function. J Neurosci 28: 6983-6995, 2008.

20. Haziza S, Magnani R, Lan D, Keinan O, Saada A, Hershkovitz E, Yanay N, Cohen Y, Nevo Y, Houtz RL, et al: Calmodulin methyltransferase is required for growth, muscle strength, somatosensory development and brain function. PLoS Genet 11: e1005388, 2015.

21. Neel BG, Gu $\mathrm{H}$ and Pao L: The 'Shp'ing news: $\mathrm{SH} 2$ domain-containing tyrosine phosphatases in cell signaling. Trends Biochem Sci 28: 284-293, 2003.

22. Xu H, Yang Y, Tang X, Zhao M, Liang F, Xu P, Hou B, Xing Y, Bao $\mathrm{X}$ and Fan X: Bergmann glia function in granule cell migration during cerebellum development. Mol Neurobiol 47: 833-844, 2013.

23. Loeb GE and Tsianos GA: Major remaining gaps in models of sensorimotor systems. Front Comput Neurosci 9: 70, 2015.

24. Dasen JS: Transcriptional networks in the early development of sensory-motor circuits. Curr Top Dev Biol 87: 119-148, 2009.

25. Roberts AE, Allanson JE, Tartaglia M and Gelb BD: Noonan syndrome. Lancet 381: 333-342, 2013.

26. Kriegstein A, Noctor S and Martínez-Cerdeño V: Patterns of neural stem and progenitor cell division may underlie evolutionary cortical expansion. Nat Rev Neurosci 7: 883-890, 2006.

27. Namba T and Huttner WB: Neural progenitor cells and their role in the development and evolutionary expansion of the neocortex. Wiley Interdiscip Rev Dev Biol 6, 2016.

28. Molyneaux BJ, Arlotta P, Menezes JR and Macklis JD: Neuronal subtype specification in the cerebral cortex. Nat Rev Neurosci 8: 427-437, 2007.

29. Sur M and Rubenstein JL: Patterning and plasticity of the cerebral cortex. Science 310: 805-810, 2005.

30. Malatesta P and Götz M: Radial glia - from boring cables to stem cell stars. Development 140: 483-486, 2013.

31. Qian X, Shen Q, Goderie SK, He W, Capela A, Davis AA and Temple S: Timing of CNS cell generation: A programmed sequence of neuron and glial cell production from isolated murine cortical stem cells. Neuron 28: 69-80, 2000.

32. Sauvageot CM and Stiles CD: Molecular mechanisms controlling cortical gliogenesis. Curr Opin Neurobiol 12: 244-249, 2002.

33. Dwyer ND, Chen B, Chou SJ, Hippenmeyer S, Nguyen L and Ghashghaei HT: Neural stem cells to cerebral cortex: Emerging mechanisms regulating progenitor behavior and productivity. J Neurosci 36: 11394-11401, 2016.

34. Guerout N, Li X and Barnabé-Heider F: Cell fate control in the developing central nervous system. Exp Cell Res 321: 77-83, 2014.

35. Lefloch R, Pouysségur J and Lenormand P: Single and combined silencing of ERK1 and ERK2 reveals their positive contribution to growth signaling depending on their expression levels. Mol Cell Biol 28: 511-527, 2008.

36. Wang VY and Zoghbi HY: Genetic regulation of cerebellar development. Nat Rev Neurosci 2: 484-491, 2001. 